\title{
SPRINGER-TYPE THEOREMS FOR SPINOR GENERA OF QUADRATIC FORMS
}

BY A. G. EARNEST AND J. S. HSIA

Communicated by D. J. Lewis, May 5, 1975

At the Quadratic Forms Conference (Baton Rouge, Louisiana, 1972), N. C. Ankeny raised the question on the behaviour of the genus of a positive definite integral quadratic form upon inflation to a totally real number field. Here we announce some results of the closely related problem of how the spinor genus behaves when lifted to an overfield. We treat this question geometrically and also in a more general setting; namely, we study the spinor genera associated with an arbitrary quadratic lattice, not necessarily a free lattice. A classical theorem of Springer [S] asserts an anisotropic quadratic space over $F$ remains anisotropic in $E$ if the field degree $[E: F]$ is odd. In terms of classical Witt rings, it says the natural map $W(F) \rightarrow W(E)$ is injective. Our results for the spinor genus behaviour are similar in spirit. Detailed proofs will appear elsewhere. Unexplained notations are from [0].

Let $E / F$ be a finite extension of global fields, $O_{E}, O_{F}$ the integers in $E, F$ respectively, $L$ a lattice on a regular space $V$ over $F$ with rank $r(L) \geqslant 3$. Put $\widetilde{V}=V \otimes E$ and $\widetilde{L}=L \otimes O_{E}$. Define maps $\beta: J_{F} \rightarrow J_{E}$ and $\gamma: J_{V} \rightarrow J_{\widetilde{V}}$, respectively, by $\left(\beta\left(j_{p}\right)\right)_{P}=j_{p}$ and $\left(\gamma\left(u_{p}\right)\right)_{P}=u_{p} \otimes E_{P}$ for $P \mid p$. Then $\beta$ and $\gamma$ induce vertical maps $\psi_{L}$ and $\Gamma_{L}$, respectively, in the commutative diagram

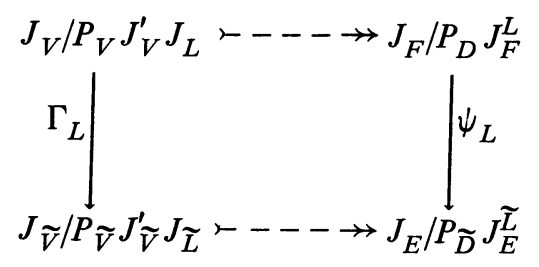

so that $\Gamma_{L}$ is injective if and only if $\psi_{L}$ is injective as the horizontal maps are isomorphisms. Our main results are:

THEOREM A. Let $L$ be a quadratic lattice of rank $r(L) \geqslant 3$ and defined over a global field $F$ with the property that at each dyadic localization $L_{p}$ is modular. Then for any odd degree field extension $E / F, \psi_{L}$ is injective.

THEOREM B. Let $L$ be a quadratic lattice of rank $r(L) \geqslant 3$. If $E / F$ is an odd degree field extension of number fields such that 2 is unramified in $E$, then $\psi_{L}$ is injective.

AMS (MOS) subject classifications (1970). Primary 10C05, 15A63; Secondary 10E45, $10 \mathrm{~B} 05,20 \mathrm{G} 30$.

Key words and phrases. Integral quadratic forms, spinor genus, quadratic lattices, spinor norms. 
REMARKS. Two important special cases where Theorem A directly applies are: (1) $F$ is a function field and so has no dyadic spots, and (2) $F=\mathbf{Q}, L$ an integral (with respect to scale) lattice with odd discriminant. When $L$ is indefinite, the well-known Eichler-Kneser theorem asserts the (proper) spinor genus and the (proper) class coincide so that Theorems A and B then say nonequivalent proper classes in a given genus remain nonequivalent when lifted to $E$. A Hasse domain is a dedekind domain with quotient field a global field $F$ and which can be obtained as the intersection of almost all valuation rings on $F$. Theorems $\mathrm{A}$ and $\mathrm{B}$ generalize to Hasse domains as well.

The proofs rely on rather long and meticulous studies of the spinor norms of integral rotations on the localizations $L_{p}$ at the nonarchimedean spots, and how these groups behave going-up and coming down. The easiest cases are when $p$ is nondyadic, where we employ a theorem of Kneser [K] who had calculated these groups at such spots. Dyadic theory is far more difficult. The conditions on Theorems A and B are such that heavy dependence is placed on the exact knowledge of $\theta\left(O^{+}\left(L_{p}\right)\right)$ when: (i) $p$ arbitrary dyadic but $L_{p}$ modular; (ii) $L_{p}$ arbitrary but $p$ unramified dyadic. Computations for (ii) are more technically involved and we derive several Kneser-type theorems (see $[\mathbf{K}]$ ). Aside from these rather intricate spinor considerations, the norm principles of Scharlau and Knebusch are needed as well as some lifting formulas for the Hilbert and Hasse symbols.

We now give examples which show the oddness of field degree is necessary in both Theorems $\mathrm{A}$ and $\mathrm{B}$.

ExAmples. (1) $F=\mathbf{Q}(\sqrt{-5}), E=\mathbf{Q}(\sqrt{-5}, \sqrt{-1})$ its Hilbert class field, $L \cong\langle 1,1,1\rangle$. One can show the number $g^{+}(L)$ of proper spinor genera in the genus of $L$ equals the order of the factor group $C_{F} / C_{F}^{2}$ where $C_{F}$ is the ideal class group of $F$. Similarly, $g^{+}(\widetilde{L})=\left|C_{E} / C_{E}^{2}\right|$. But, $C_{E}$ is trivial. Hence, $\psi_{L}$ must collapse. (2) $F=\mathrm{Q}, E=\mathrm{Q}(\sqrt{5}), L \cong\left\langle-1,5^{2} 13^{2}, 5^{4} 13^{4}\right\rangle$. Then, $\theta\left(O^{+}\left(L_{p}\right)\right)=$ $Q_{2}^{*}$ for $p=2 ;=Q_{5}^{* 2}, Q_{13}^{* 2}$ for $p=5,13$ respectively; and $=U_{p} Q_{p}^{* 2}$ for all other $p$ 's. Using these one shows $g^{+}(L)=2$. While $g^{+}(\widetilde{L})=2$ also, $\psi_{L}$ is not injective. Note that we may use the definite lattice $K \cong\left\langle 1,5^{2} 13^{2}, 5^{4} 13^{4}\right\rangle$ and also conclude that $\psi_{K}$ is not injective.

\section{REFERENCES}

[K] M. Kneser, Klassenzahlen indefiniter quadratischer Formen in drei oder mehr Veränderlichen, Arch. Math. 7 (1956), 323-332. MR 18, 562.

[O] O. T. O'Meara, Introduction to quadratic forms, Die Grundlehren der math. Wissenschaften, Band 117, Academic Press, New York; Springer-Verlag, Berlin, 1963. MR 27 \#2485.

[S] T. A. Springer, Sur les formes quadratiques d'indice zéro, C. R. Acad. Sci., Paris 234 (1952), 1517-1519. MR 13, 815.

DEPARTMENT OF MATHEMATICS, OHIO STATE UNIVERSITY, COLUMBUS, 